\title{
Error Control Codes for Next-Generation Communication Systems: Opportunities and Challenges
}

\author{
Zesong Fei $\mathbb{D}^{1},{ }^{1}$ Jinhong Yuan, ${ }^{2}$ and Qin Huang $\mathbb{D}^{3}$ \\ ${ }^{1}$ School of Information and Electronics, Beijing Institute of Technology, Beijing 10081, China \\ ${ }^{2}$ School of Electrical Engineering and Telecommunications, University of New South Wales, Sydney, NSW 2052, Australia \\ ${ }^{3}$ School of Electronic and Information Engineering, Beihang University, Beijing 100191, China \\ Correspondence should be addressed to Zesong Fei; feizesong@bit.edu.cn
}

Received 3 October 2018; Accepted 3 October 2018; Published 2 December 2018

Copyright (c) 2018 Zesong Fei et al. This is an open access article distributed under the Creative Commons Attribution License, which permits unrestricted use, distribution, and reproduction in any medium, provided the original work is properly cited.

Error control codes are widely applied in modern communication systems to improve the bandwidth-power efficiency and the reliability of data transmissions. Modern error control codes have attracted the interest of scholars and industry partners since Turbo codes were invented. For example, Turbo codes have been used in the $4 \mathrm{G}$ cellular mobile systems. Nowadays, LDPC codes and the polar codes are adopted in the $5 \mathrm{G}$ standard. The recent development on the theoretic framework of new channel coding theorem for finite code length will provide guidelines for future practical error control codes designs.

In the age of IoT, everything will be connected via communication links. It is expected that the next-generation communication systems need to support many scenarios such as wireless communications, optical communications, distributed storage systems, V2X networks, and sensor networks. These scenarios will impose new requirements to the communication systems ranging from lower complexity encoder/decoder, lower delay or latencies, ultrareliable transmission at rates close to the Shannon capacity, low energy consumptions, etc. In addition to the communication systems, error control codes also find emerging applications in security, flash memories, and deep-space probing.

This special issue is a collection of 11 papers which explore the performance of error control codes for the next generation communication systems and discuss the opportunities and challenges that they will face.
"Superposition Coded Modulation Based Faster-ThanNyquist Signaling", by S. Li et al., presented a transmission scheme of faster-than-Nyquist signaling combined with superposition coded modulation. The proposed scheme requires a lower SNR than orthogonal signaling with a larger alphabet to achieve a wide range of spectral efficiencies.

"A Novel Design of Downlink Control Information Encoding and Decoding Based on Polar Codes", by C. Sun et al., proposed a novel design on downlink control information encoding and decoding. The proposed scheme could support dynamic configuration of transmission modes with low complexity. It is shown that the proposed scheme can comply with the false alarm rate target of 5G standard.

"Performance Analysis of CRC Codes for Systematic and Nonsystematic Polar Codes with List Decoding", by T. Murata and H. Ochiai, studied the effect of the length and generator polynomials of CRC codes on frame error rate performance of polar codes with list decoding. The authors found that the length of CRC will affect the performance significantly, while the generator polynomials will only affect nonsystematic polar codes.

"Adding a Rate-1 Third Dimension to Parallel Concatenated Systematic Polar Code: 3D Polar Code", by Z. Liu et al., proposed a three-dimensional polar code scheme to improve the error floor performance of parallel concatenated systematic polar codes. The key idea of the proposed scheme is that it makes the best use of the characteristic of parallel concatenation and serial concatenation. 
"Construction and Decoding of Rate-Compatible Globally Coupled LDPC Codes", by J. Zhang et al., presented a family of rate-compatible globally coupled LDPC codes which provide more flexibility in code rate and guarantee the structural property of algebraic construction. The authors also proposed a modified low complexity decoding scheme.

"Construction of Quasi-Cyclic LDPC Codes Based on Fundamental Theorem of Arithmetic", by H. Zhu et al., studied the construction of Quasi-Cyclic LDOC codes based on an arbitrary given expansion factor. The constructed codes perform well in AWGN channels when iterative decoding algorithms are used.

"Code-Hopping Based Transmission Scheme for Wireless Physical-Layer Security", by L. Yin and W. Hao, proposed a code-hopping based secrecy transmission scheme that uses dynamic nonsystematic LDPC codes and automatic repeatrequest $(A R Q)$ mechanism to jointly encode and encrypt source messages. In this scheme, source message is jointly encoded and encrypted by a parity-check matrix which is dynamically selected from a set of LDPC matrices based on the shared secret key. The authors showed that the key is difficult for the eavesdropper to generate, and the security gap is small.

"Research and Implementation of Rateless Spinal Codes Based Massive MIMO System”, by L. Wang et al., proposed a spinal codes-aided massive MIMO scheme and a multilevel puncturing and dynamic block-size allocation scheme. The proposed schemes can approach the maximum achievable rate, and a comparable MIMO testbed is established to demonstrate the effectiveness of the proposed scheme.

"Design and Analysis of Adaptive Message Coding on LDPC Decoder with Faulty Storage", by G. G and L. Yin, discussed the impacts of message errors on LDPC decoders with unreliable memories. An adaptive coding scheme based on the magnitude level of messages was also proposed to improve the robustness.

"A CCM-Based OFDM System with Low PAPR for Sparse Source", by Q. Yang et al., introduced compressive coded modulation scheme to restrain peak-to-average power ratio in OFDM systems, which becomes severe when the source is sparse.

"Efficient Quantization with Linear Index Coding for Deep-Space Images", by R. Mahmood et al., proposed a modified quantization with a linear index coding scheme to improve its spectral efficiency and robustness. The proposed scheme efficiently removes the redundant bit-planes for spectrally efficient linear index coding of images. A multipass decoding scheme is also proposed which provides better gain by using extrinsic information.

\section{Conflicts of Interest}

The authors declare that there are no conflicts of interest regarding the publication of this article.

\section{Acknowledgments}

The editors thank all of the authors for their submissions to this special issue. We are also grateful to the anonymous reviewers for their valuable comments to improve the quality of the articles. We hope that this special issue will further encourage research interests and engineering practice in the area of Error Control Codes.

Zesong Fei

Jinhong Yuan

Qin Huang 


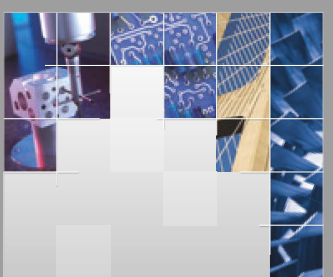

\section{Enfincering}
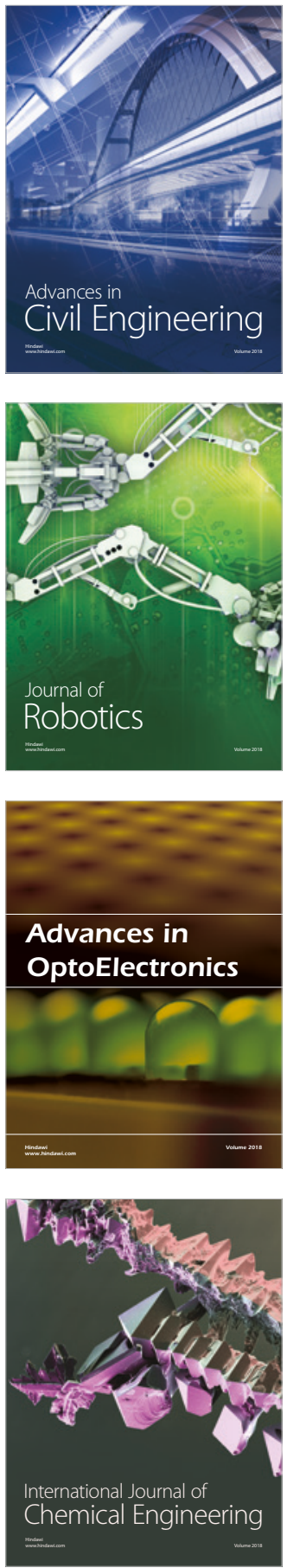

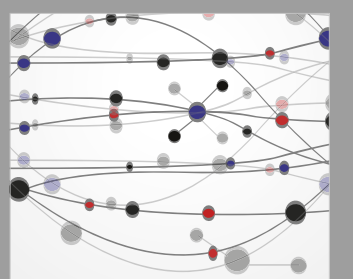

\section{Rotating \\ Machinery}

The Scientific World Journal

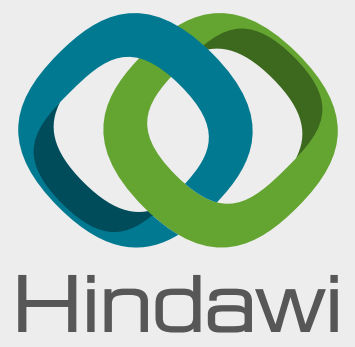

Submit your manuscripts at

www.hindawi.com
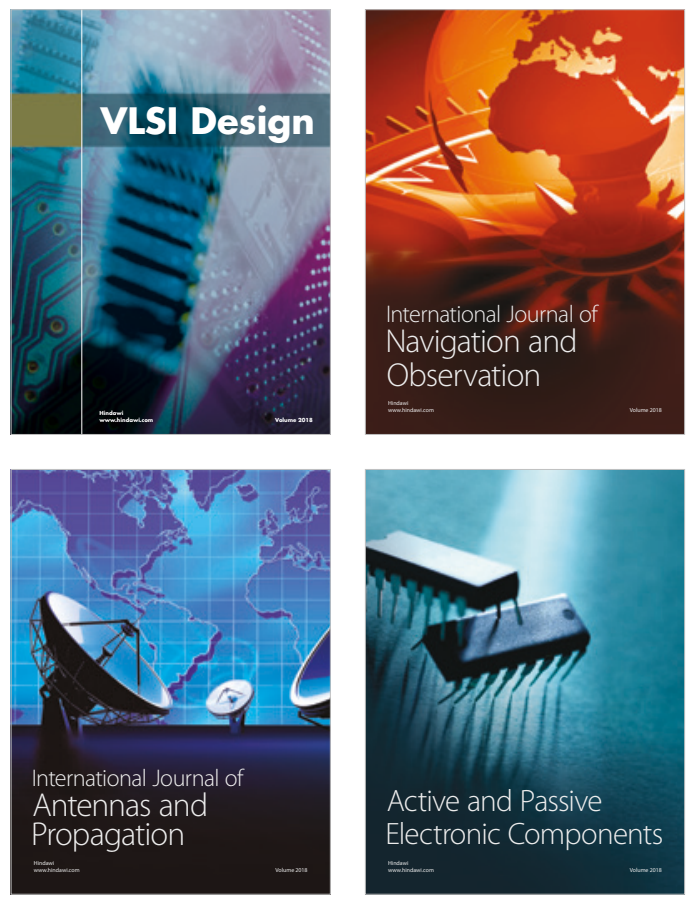
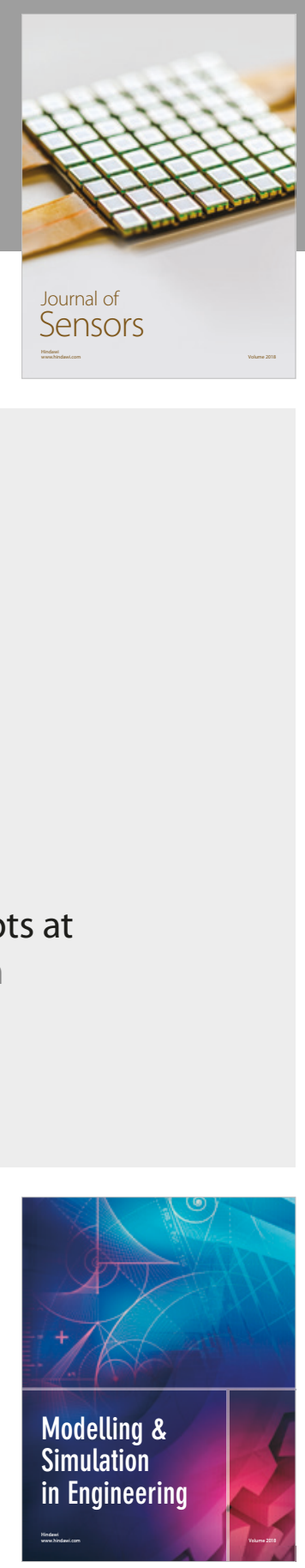

\section{Advances \\ Multimedia}
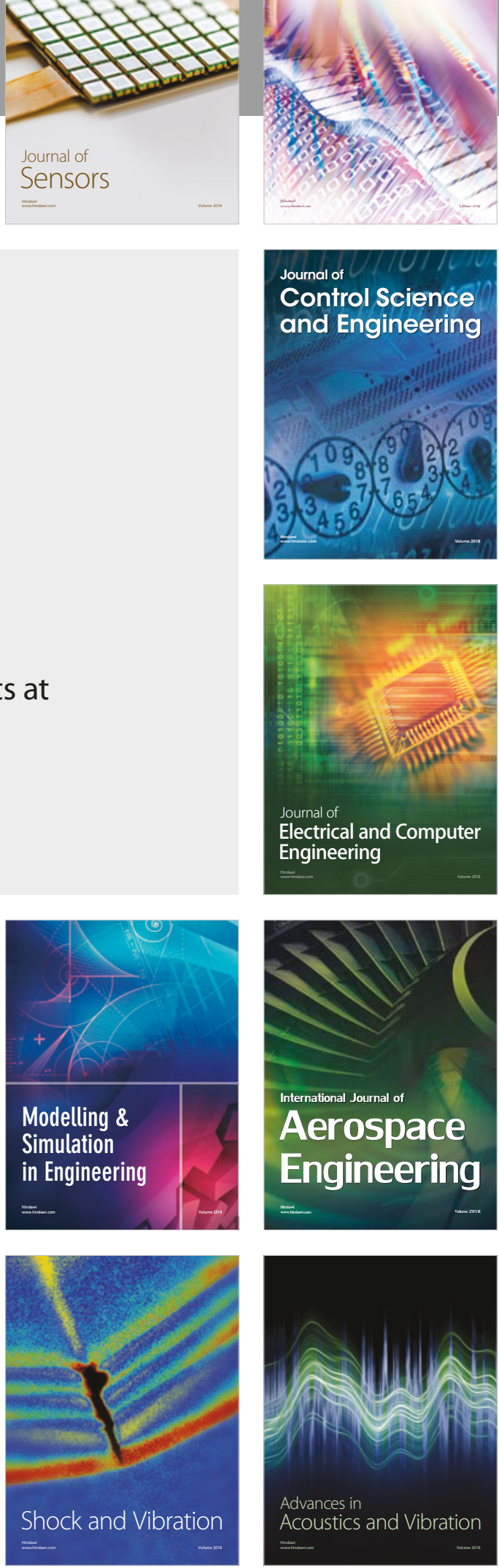